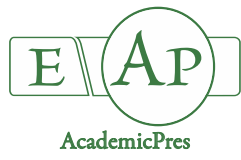

\title{
Development of an Efficient 'One-Step Freezing' Cryopreservation Protocol for a Georgian Provenance of Chestnut (Castanea sativa Mill.) Zygotic Embryos
}

\author{
Mariam GAIDAMASHVILI ${ }^{1}$, Eka KHURTSIDZE ${ }^{1}$, Carla BENELLI², \\ Maurizio LAMBARDI ${ }^{2 *}$ \\ ${ }^{1}$ Iv. Javakhishvili Tbilisi State University, Faculty of Exact and Natural Sciences, Department of Biology, 1, Chavchavadze Ave. 0179, Tbilisi, \\ Georgia; mariam.gaidamashvili@tsu.ge; ekakhurtsidze@tsu.ge \\ ${ }^{2}$ National Research Council (CNR), IBE/Institute of BioEconomy, via Madonna del Piano 10, 50019 Sesto Fiorentino, Firenze, \\ Italy; carla.benelli@cnr.it;maurizio.lambardi@cnr.it (*correspondingauthor)
}

\begin{abstract}
Experiments were performed to determine the influence of various dehydration and vitrification treatment times on the 'one-step freezing' cryopreservation of embryonic axes (EAs), composed of zygotic embryos and cotyledon residuals, from mature seeds of a Georgian provenance of chestnut (Castanea sativa Mill.). Dehydration was carried out in laminar flow hood from 1 to $5 \mathrm{~h}$, and vitrification experiments were carried out by immersion of EAs in PVS2 vitrification solution up to 120 $\mathrm{min}$, both followed by direct immersion in liquid nitrogen. Both systems resulted in inducing specimen tolerance to ultra-rapid freezing, although to a different extent. Full germination of cryo-stored EAs after $5 \mathrm{~h}$ of dehydration (reducing moisture content from initial $66 \%$ to $21 \%$ ) has been increased from $0 \%$ to $66.7 \%$. A pre-treatment of EAs in PVS2 vitrification solution for $30 \mathrm{~min}$ produced fully developed plantlets at a rate of $55.6 \%$ in post-cryopreservation. Plantlet regrowth from cryopreservation was faster in EAs that underwent the dehydration/'one-step freezing' procedure. All the plantlet from cryopreserved EAs could be easily acclimatized, producing healthy potted plants. Finally, the TTC test showed to be useful for a fast evaluation of specimen survival after thawing and, as a consequence, to speed up the development of optimized cryoprotocols.
\end{abstract}

Keywords: chestnut; cryopreservation; dehydration; embryonic axes; vitrification; zygotic embryos

\section{Introduction}

The forest ecosystems are of special significance for conservation of biodiversity of Georgia. The forest covers $39.9 \%$ of the territory of the country. Their greater percentage $(98 \%)$ is presented by mountainous forests and they have water regulating, soil protective, climate stabilizing functions. Besides, they are an important location of many relict, endemic and threatened species of plants and animals. European chestnut or sweet chestnut (Castanea sativa Mill.) is the dominant of mountainous forests of Western Georgia (150-1800 m). It occupies the most percentage of areas covered with forests (approx. 75\%). Dendroflora of Caucasus, issued earlier in 1961, lists 14 species within genus Castanea; however, only Castanea sativa Mill. occurs in Georgia (Gulisashvili, 1961). Chestnut forests are distributed continuously along the southern slope of the Caucasus Mountains near the Black Sea and are found in isolated populations on the north side of the Caucasus, at elevations ranging from 200 to 1400 meters (Pridnya et al., 1996). Chestnut-dominated forests comprise only a few percent of total forest cover in the Caucasus Biosphere Preserve, and usually occur in mountain valleys or coves with deep brown soil. Castanea sativa forests are developed in both West and East Georgia, but to the west of the country they occupy larger areas. In some localities pure stands of $C$. sativa can be found, but mainly sweet chestnut occurs as a component of oligodominant beech-sweet chestnut and hornbeam-beech-sweet chestnut forests (Gagnidze, 1975). Chestnut trees generally extend from 200 (West Georgia) up to 900-1000 meters above sea level, having the absolute upper limit at 1400 meters in sporadic locations of West and East Georgia (Nakhutsrishvili, 2013).

At present, due to low self-renewal and diseases, the big massifs of chestnut forests in Caucasus are on the verge of 
1048

destruction. Chestnut blight was apparently introduced into the region after 1880 and continues to destroy chestnut forests today. Although chestnut seed production appears adequate, the competition with shrubs and other tree seedlings, as well as predation by herbivores and rodents, interrupts the establishment and survival of chestnut seedlings in the biosphere preserve. In addition, sweet chestnut in the Caucasus is infested by several fungal and bacterial parasites (Tavadze et al., 2012). Castanea sativa Mill. is filed into the Red List of Georgia (2006) due to the tendency to the decrease of the distribution range and habitat fragmentation. IUCN category for this taxon is evaluated as Vulnerable (VU), according to the IUCN Red List Categories and Criteria: population reduction observed, estimated, inferred, or suspected in the past where the causes of reduction may not have ceased or may not be understood or may not be reversible. According to the official IUCN list (2019) Castanea sativa has been assessed as Least Concern (Barstow and Khela, 2018).

The seeds of Castanea sativa are classified as recalcitrant (Pence, 1992; Westengen et al., 2013). In contrast to orthodox seeds, the recalcitrant seeds are shed at high water contents and are highly susceptible to desiccation and sometimes also to low temperatures (Vertucci and Farrant, 1995; Berjak and Pammenter, 1997). Such behaviour poses a major problem to seed banks for long-term conservation. Axes and cotyledons of recalcitrant seeds of Castanea sativa Mill. exhibit contrasting responses of respiration and sensitivity in relation to desiccation (Leprince et al., 1999), and their conservation at low above-freezing $\left(0-2{ }^{\circ} \mathrm{C}\right)$ temperature (Giacalone and Bounous, 1993) allows the maintenance of seed viability and germinability only up to 90 days (Conedera et al., 2004). This makes urgent search for alternative techniques for conservation of this vulnerable taxon.

Conservation of shoot cultures at low above-freezing temperature is one option for ex situ medium-term preservation of woody plant germplasm. As for chestnut, the technique has been investigated, allowing the conservation of micropropagated shoot cultures for a limited period of time at $4^{\circ} \mathrm{C}$ (Lambardi $e t$ al., 2001), and at $8{ }^{\circ} \mathrm{C}$ (Capuana and Di Lonardo, 2013). Cryopreservation or freeze-preservation at the ultra-low temperature $(-196$ ${ }^{\circ} \mathrm{C}$ ) of liquid nitrogen (LN) is the only technique currently available to ensure the safe and cost-efficient long-term conservation of germplasm of both seed and vegetativelypropagated species (Panis and Lambardi, 2006; Benelli et al., 2013). It is of particular practical interest for the long-term conservation of species characterized by sub-orthodox and non-orthodox seeds (Pritchard and Nadarajan, 2008; Walters et al., 2013). At the temperature of LN, biochemical and most physical processes are completely arrested. In this context, cryopreservation should be regarded as a sound complementary approach to the traditional seed- and in-field bank strategy of germplasm preservation, providing a real guarantee against accidental loss of plant genetic resources. As for chestnut, few reports are available, regarding the conservation in LN of shoot tips (San-José et al., 2005; Vidal et al., 2005; Vidal et al. 2010), zygotic embryo axes (Corredoira et al., 2004; San-José et al., 2005), and somatic embryos (San-José et al., 2005; Vidal et al., 2010). However, such reports always refer to seeds collected from local open-pollinated chestnut trees, and the wide applicability of selected protocols to germplasm from different and distant (in terms of geographical areas and climatic characteristics) provenances has never tested.

In order to develop an efficient strategy for the longterm conservation of threatened chestnut Georgian provenances, the present study developed a step-wise 'onestep freezing' protocol for the cryopreservation of excised embryonic axes (EAs) from the Caucasus region, comparing two different cryo-techniques, i.e., specimen dehydration and PVS2-vitrification, both followed by the direct immersion of EAs in LN ('one-step freezing'). Moreover, the application of a test of seed viability after their recover from LN has been applied for the first time in chestnut, with the aim to speed up the optimization of an efficient and repetitive cryo-procedure.

\section{Materials and Methods}

\section{Biologicalmaterial}

Castanea sativa hedgehogs were collected in OctoberNovember 2018 from Tkibuli region in western Georgia, Imereti, at an altitude of 600-800 meters above sea level. After extraction from the hedgehogs, mature fruits were stored at $4{ }^{\circ} \mathrm{C}$ for a maximum of $45^{\circ}$ days, until use in the cryopreservation trials.

\section{Dehydration/'one step freezing'}

For cryopreservation experiments, the fruit were washed in household detergent (2\%) and rinsed three times under tap water. Then the pericarp, seed coat and lateral part of endosperm were removed. The remaining seeds were surface sterilized by successive immersion in $70 \%(\mathrm{v} / \mathrm{v})$ ethanol with few drops of Tween 20 for $2 \mathrm{~min}$, followed by decontamination with $10 \%$ solution of sodium hypochlorite for $20 \mathrm{~min}$. After being rinsed in sterile distilled water tree times, EAs, composed by the zygotic embryos along with cotylenodary knots and 2-3 mm-long cotyledon residuals, were dissected from seeds (Fig. 5A).

To determine the moisture content (MC) of EAs, the fresh weight of single EAs, with 5 replicates for each desiccation time, was measured. After final period of desiccation time, embryos were dried at $85^{\circ} \mathrm{C}$ for $24 \mathrm{~h}$ and re-weighted. MC was calculated as a percentage of embryo fresh weight.

As for cryopreservation trials, for each of 5 desiccation times $(0,1,2,3,4$, and $5 \mathrm{~h}), 50$ EAs were desiccated in open Petri dishes under the air flux of a laminar flow hood. Thirty specimen were then used for determination of MC (10 EAs), for the tetrazolium chloride (TTC) test (see below; 10 EAs), and for germinability determination (10 EAs) after dehydration but without immersion in LN (LN-). Twenty EAs $(10$ for viability, and 10 for germinability determination) were placed in 2-ml cryovials (10 EAs in each) and immersed in LN for $24 \mathrm{~h}(\mathrm{LN}+)$. For thawing, the cryovials were rapidly immersed in water bath at $40{ }^{\circ} \mathrm{C}$ for $2 \mathrm{~min}$. As for EA germinability (for its determination, see below), LN+ and LN- samples were cultured in WPM (Lloyd and McCown, 1981) with 0.4 $\mu \mathrm{M}$ 6benzylaminopurine (BAP) and $0.3 \%$ activated charcoal 
(Sigma-Aldrich), inside test tubes $(20 \mathrm{~mm} \times 150 \mathrm{~mm})$. Sucrose was supplied at $30 \mathrm{~g} \mathrm{l}^{-1}$. The media were then solidified with $6 \mathrm{~g} \mathrm{l}^{-1}$ agar and adjusted to $\mathrm{pH} 5.7$ before autoclaving. EAs were maintained in growth chamber at $24 \pm 0.5^{\circ} \mathrm{C}$ under $16 / 8 \mathrm{~h}$ photoperiod with an irradiance of $40 \mu \mathrm{mol} \mathrm{m} \mathrm{s}^{-1}$ from cool-white fluorescent tubes.

\section{PVS2-vitrification/'one step freezing'}

EAs were transferred to loading solution (LS) containing $2 \mathrm{M}$ glycerol and $0.4 \mathrm{M}$ sucrose for $20 \mathrm{~min}$ at 24 ${ }^{\circ} \mathrm{C}$. Then, the EAs were placed in 2-ml cryovials and treated with PVS2 vitrification solution (30\% w/v glycerol, $15 \%$, $\mathrm{w} / \mathrm{v}$ DMSO, $15 \% \mathrm{w} / \mathrm{v}$ ethylene glycol in WPM containing $0.4 \mathrm{M}$ sucrose; Sakai et al., 1990), for different times (0, 30, $60,90,120 \mathrm{~min}$ ) at $0^{\circ} \mathrm{C}$, after they were plugged in $\mathrm{LN}$ for $24 \mathrm{~h}$. For each treatment time, 40 embryos were used. A control without any treatment and LN- (10 EAs), was also included. For 0-min treatment time, EAs were only loaded in LS solution and then cultured in test tubes for germination. After immersion in $\mathrm{LN}$ the cryovials were thawed in a water bath at $40^{\circ} \mathrm{C}$ for $2 \mathrm{~min}$, and the EAs were rinsed in washing solution containing WPM liquid medium and $1.2 \mathrm{M}$ sucrose (two times for $10 \mathrm{~min}$ each, at $25^{\circ} \mathrm{C}$ ), and then cultured in test tubes, as reported above.

Determination of EAs viability after cryopreservation by the TTC test

The viability of EAs was evaluated using $1 \%$ TTC solution. Ten EAs for each dehydration time followed by cryopreservation, and 10 dehydrated but not cryopreserved (LN-), were transferred on filter paper in $90 \mathrm{~mm}$ Petri dishes. The EAs were covered with the TTC solution and placed overnight in the dark at $20^{\circ} \mathrm{C}$. After staining, the solution was drained off and EAs were rinsed under tap water. Viability of each EA was interpreted according to the topographical staining pattern of the embryo and the intensity of the coloration with the help of magnifying glass. EAs were identified as 'fully viable' when embryo axes and cotyledon residuals were fully stained. Completely unstained EAs or stained in small patches were evaluated as 'non-viable'.

\section{Data collection and statistical analysis}

The total number of EAs used was 300 for dehydration/'one-step freezing', and 240 for PVS2vitrification/'one-step freezing'. Each treatment consisted in 3 replicates and all experiments were repeated 3 times. EAs germinability was assessed first time after 7 days of culture, reported as 'survival' that indicated the percentage of EAs showing initial root and shoot emission after re-culturing (early germinability). No all surviving EAs showed subsequent development and, after 4 (dehydration/'one- step freezing') or 8 (PVS2-vitrification/'one-step freezing') weeks of culture, plantlet regrowth rate was estimated as the percentage of fully developed plantlets (full germinability), retaining normal shoots and roots (roots and shoots $\geq 5$ $\mathrm{mm})$.

Statistical analysis was performed by ANOVA, followed by mean comparison using the LSD (Least Significant Difference) test at $\mathrm{p} \leq 0.05$ level. Percentage data were subjected to arcsine transformation prior to analysis. Bars represent standard errors (SE) of means.

\section{Results}

Cryopreservation by dehydration/'one-step freezing' of $E A$ s The initial MC of Castanea sativa EAs from seeds stored one month at $4{ }^{\circ} \mathrm{C}$ was $66.2 \%$ respect to fresh weight (Table 1; $0 \mathrm{~h}$ dehydration). A decrease of $\mathrm{MC}$ was then observed after each time of dehydration under the sterile air of laminar hood, significative starting from $2 \mathrm{~h}$ of dehydration and reaching a final of $21.4 \%$ of $\mathrm{MC}$ after 5 hours (Fig. 1A and $\mathrm{B})$. This dehydration trend of EAs that were not cryopreserved (LN-) didn't affect the survival of EAs which remained 100\% even at the lowest MC (Fig. 1A); however, not all these EAs completed the germination process, as plantlet regrowth from EAs dehydrated for 5 hours was reduced to $84 \%$. EAs which were directly immersed in $\mathrm{LN}$, without any previous dehydration (time 0 ), never survived to cryopreservation. The dehydration procedure, on the other hand, induced a clear effect of tolerance of the EAs to ultra-rapid freezing in LN. Indeed, even after only $1 \mathrm{~h}$ of dehydration, EAs with a MC reduced to $62.8 \%$ showed a $21.4 \%$ survival to LN. Increasing the dehydration time produced a parallel increase in the survival of EAs which was significative starting from 3-h dehydration and that reached a maximum of $80 \%$ after $5 \mathrm{~h}$.

The survival of EAs refers to the first clear signs of germination (see 'Material and Methods'), followed by shoot elongation (Fig. 5B). However, part of the apparently survived EAs did not evolved in plantlets (Fig. 1B), showing only root elongation (Fig. 5C). First evidences of plantlet regrowth (21.8\%) was achieved in EAs that were dehydrated for at least $2 \mathrm{~h}$, producing a reduction of their $\mathrm{MC}$ to $41.2 \%$. Increasing the time of dehydration up to $5 \mathrm{~h}$ induced a constant increase of plantlet regrowth, up to a maximum of $66.7 \%$, a value significantly higher than the ones achieved with lower dehydration times (Fig. 5D). Afterwards, plantlets were easily transferred in pots and acclimatated (Fig. 5E).

The TTC test showed to be an effective test for a fast evaluation of EAs viability following cryopreservation. Comparing the two lines reported in Fig. 2, the 'viability' line (expressing the percentages of EAs showing full red

Table 1. Plantlet regrowth of Castanea sativa EAs subjected to various dehydration times with (LN+) or without (LN-) immersion in LN, evaluated 4 weeks after cryostorage, thawing and plating (mean values $\pm \mathrm{SE}$ )

\begin{tabular}{cccccccc}
\hline Dehydration time $(\mathrm{h})$ & & 0 & 1 & 2 & 3 & 4 \\
\hline \multirow{2}{*}{ Root length $(\mathrm{mm})$} & $\mathrm{LN}-$ & $54.6 \pm 2.0$ & $48.8 \pm 0.6$ & $40.1 \pm 0.8$ & $43.8 \pm 1.2$ & $52.2 \pm 2.1$ & $49.7 \pm 1.7$ \\
& $\mathrm{LN}+$ & - & $5.7 \pm 0.3$ & $5.0 \pm 0.4$ & $6.0 \pm 0.3$ & $8.0 \pm 0.2$ & $40.1 \pm 0.2$ \\
\hline \multirow{2}{*}{ Shoot length $(\mathrm{mm})$} & $\mathrm{LN}-$ & $33.0 \pm 1.4$ & $31.8 \pm 1.2$ & $28.4 \pm 1.1$ & $23.2 \pm 1.3$ & $29.8 \pm 1.9$ & $23.0 \pm 1.4$ \\
& $\mathrm{LN}+$ & - & - & $5.0 \pm 0.4$ & $5.8 \pm 0.2$ & $7.13 \pm 0.7$ & $22.2 \pm 0.4$ \\
\hline
\end{tabular}


1050

colour after recovery from LN and thawing) has a similar pattern to the 'survival' line, with a gap between the two lines of about $40 \%$ up to $4 \mathrm{~h}$ dehydration. Following a $5 \mathrm{~h}$ dehydration of EAs, the gap between the two lines was $20 \%$.

It is interesting to note that the further development and plantlet regrowth in post-cryopreservation $(\mathrm{LN}+)$ of EAs dehydrated $5 \mathrm{~h}$ was much faster than EAs subjected to lower dehydration times, showing after 4 weeks roots longer than $4 \mathrm{~cm}$ and shoots longer than $2 \mathrm{~cm}$ (Table 1).
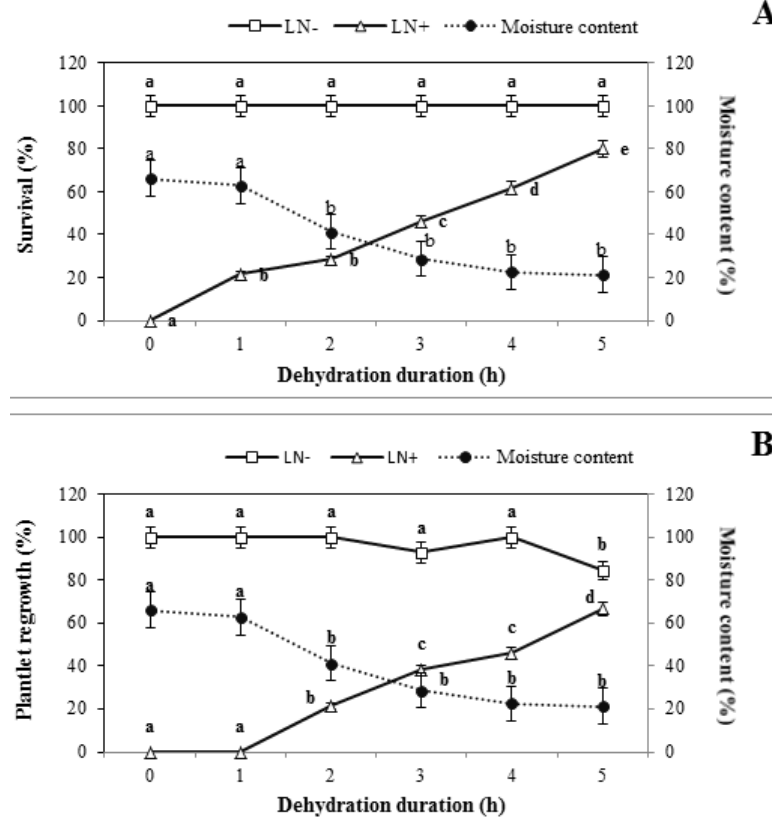

Fig. 1. Percentages of moisture content, survival (A) and plantlet regrowth (B) of Castanea sativa EAs from Tkibuli region, following increasing dehydration times with $(\mathrm{LN}+)$ or without (LN-) cryopreservation. Within each line, percentage values followed by the same letter are not significantly different at the $\mathrm{P} \leq 0.05$ by ANOVA and LSD test (bars, SE of means)

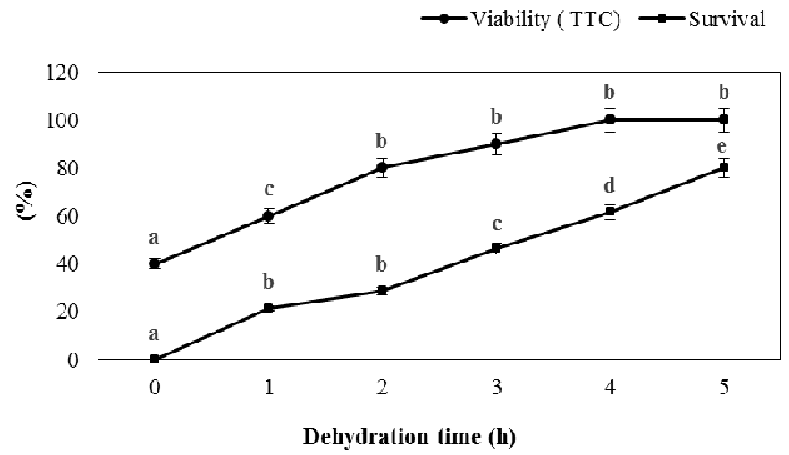

Fig. 2. Comparison between the viability, as resulted by the TTC test, and actual survival of dehydrated and cryopreserved EAs. Within each line, percentage values followed by the same letter are not significantly different at the $\mathrm{P} \leq 0.05$ by ANOVA and LSD test

\section{Cryopreservation by PVS2-vitrification of EAs}

The LS, containing $2 \mathrm{M}$ glycerol and $0.4 \mathrm{M}$ sucrose, showed to have a limited toxic effect on the EAs (Fig. 3A). Indeed, the treatment with only LS for $20 \mathrm{~min}$ at $0{ }^{\circ} \mathrm{C}$, induced a small reduction of survival from $100 \%$ (control, non-treated and non-cryopreserved) to $83.4 \%$ (time 0 ), although significant with the untreated/LN- control. However, it induced also a tolerance to ultra-rapid freezing in LN, as EAs survival and plantlet regrowth passed from nil to almost 20\%. When the EAs, following the LS treatment, were then treated with the PVS2 vitrification solution for increasing period of time and non-cryostored (LN-), EAs survival remained in the range of $75 \%$ (30 and $60 \mathrm{~min}$ of treatment) and $83.3 \%$ (90 and $120 \mathrm{~min}$ ), which never significant differences of percentage values. Referring to EAs that were afterwards cryopreserved (LN+), a 30-min treatment with PVS2 showed to produce best result in terms of EAs survival (55.6\%), significatively different from percentages obtained with both lower and higher PVS2 treatments. Indeed, when the exposure to PVS2 was increased to $60 \mathrm{~min}$, a drastic decline of survival after immersion in LN and thawing was observed (25\%) and reached a minimum of $20 \%$ following a PVS2 treatment of $120 \mathrm{~min}$

The treatment with PVS2 showed to negatively affect the further EAs development and regrowth in plantlets, as non-cryostored EAs (LN-) developed in plantlets from a maximum of $66.7 \%$ (30 min of PVS2 treatment) to a minimum of $40.2 \%$ (120 min of PVS2 treatment) (Fig. 3B). As for cryopreserved EAs (LN+), differently from the dehydration procedure, all the EAs evolved in plantlets, confirming that the 30 -min PVS2 treatment was the most effective in inducing partial tolerance to ultra-rapid freezing in LN.

The TTC test had a partially different trend from the application to the dehydration/'one-step freezing' procedure (Fig. 4). Indeed, starting from a PVS2-treatment time of $30 \mathrm{~min}$, the overestimation of the TTC test was always in the range of $40-60 \%$, being $40 \%$ (similarly to the value observed following the dehydration procedure) at the best treatment time of $30 \mathrm{~min}$.

Full germination of EAs (expressed as plantlet regrowth) required a double time ( 8 weeks), in comparison to the dehydration/'one-step freezing' procedure. Similarly to what observed with the latter procedure, plantlet regrowth was faster in EAs that were PVS2-treated with the time achiving best result in terms of sample survival. Indeed, EAs, PVS2-treated for $30 \mathrm{~min}$ and cryopreserved, showed in post-cryopreservation root and shoot regrowth over $2 \mathrm{~cm}$ after 8 weeks from thawing and plating (Table 2).

\section{Discussion}

In the past 30 years, various explants (tissue and organs) have been succesfully used in plant cryopreservation (Panis and Lambardi, 2006; Reed, 2008). Among them, sound cryopreservation procedures have been developed for orthodox (Pritchard and Nadarajan, 2008) and nonorthodox (Walters et al., 2013) by means of the storage in LN both entire seeds and excised embryonic axes (Normah and Makeen, 2008). Cryopreservation of entire seeds sometimes does not require any pre-treatment to induce 
Table 2. Plantlet regrowth of Castanea sativa EAs subjected to various PVS2-treatment times with (LN+) or without (LN-) immersion in LN, evaluated 8 weeks after cryostorage, thawing and plating (mean values $\pm \mathrm{SE}$ )

\begin{tabular}{cccccccc}
\hline PVS2-vitrification time (min) & & Control & 0 & 30 & 60 & 90 & 120 \\
\hline \multirow{2}{*}{ Root length (mm) } & LN- & $66.5 \pm 0.7$ & $20.0 \pm 0.8$ & $36.3 \pm 1.2$ & $10.7 \pm 0.3$ & $26.3 \pm 1.8$ & $24.7 \pm 0.5$ \\
& LN+ & - & $5.0 \pm 0.2$ & $27.4 \pm 0.2$ & $8.3 \pm 0.3$ & $7.9 \pm 0.4$ & $9.9 \pm 0.4$ \\
\hline \multirow{2}{*}{ Shoot length (mm) } & LN- & $42.0 \pm 1.4$ & $30.0 \pm 0.6$ & $30.0 \pm 0.6$ & $21.7 \pm 1.1$ & $26.0 \pm 0.4$ & $24.4 \pm 1.3$ \\
& LN+ & - & $4.0 \pm 0.1$ & $23.4 \pm 0.3$ & $11.2 \pm 0.2$ & $9.7 \pm 0.2$ & $6.7 \pm 0.2$ \\
\hline
\end{tabular}
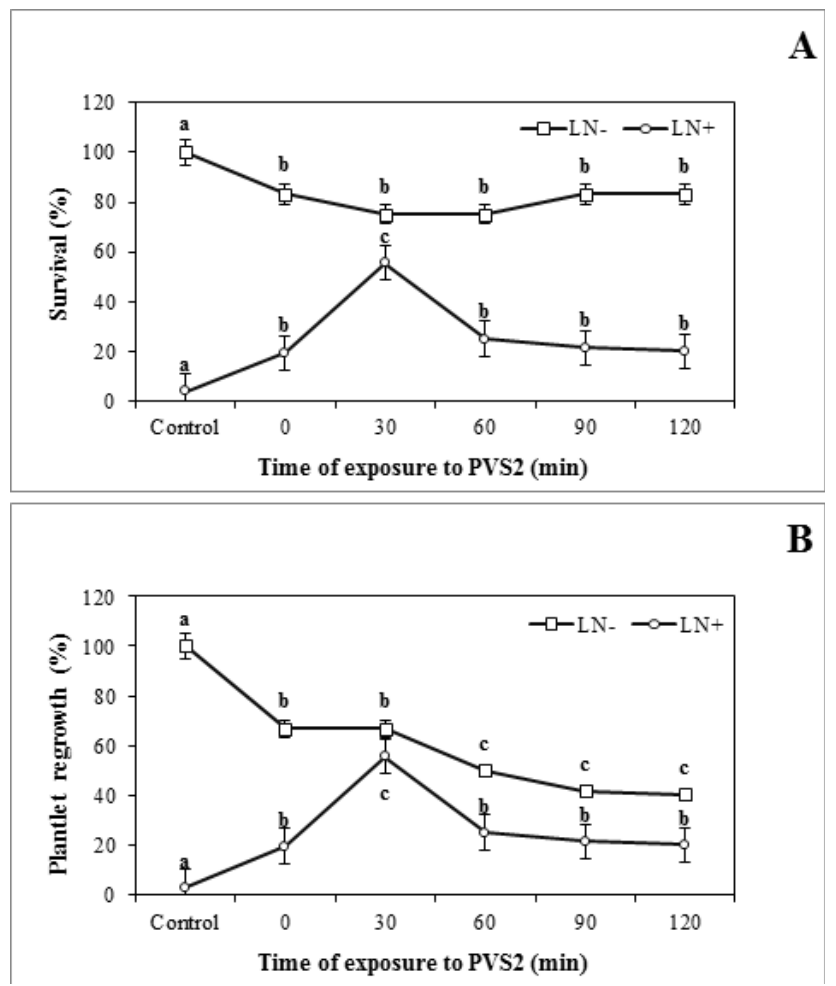

Fig. 3. Percentages of moisture content, survival (A) and plantlet regrowth (B) of Castanea sativa EAs from Tkibuli region, following increasing PVS2 treatment times with $(\mathrm{LN}+)$ or without (LN-) cryopreservation. Within each line, percentage values followed by the same letter are not significantly different at the $\mathrm{P} \leq 0.05$ by ANOVA and LSD test (bars, SE of means)

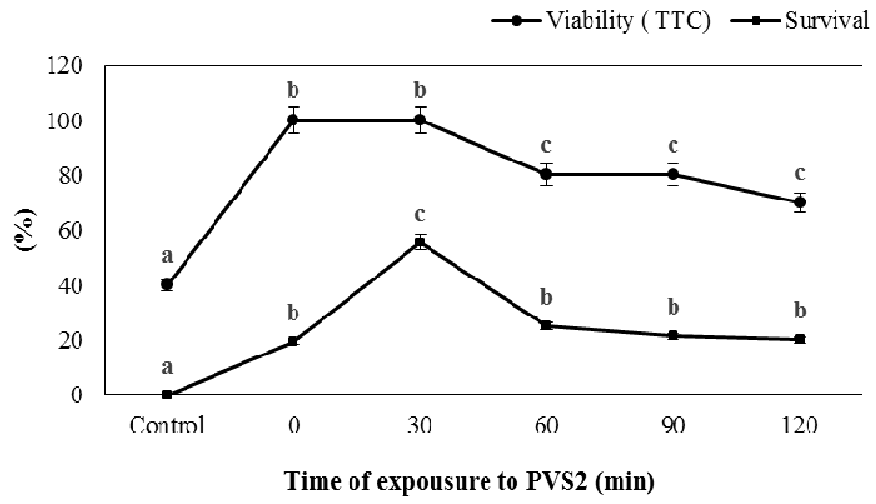

Fig. 4. Comparison between the viability, as resulted by the TTC test, and actual survival of PVS2-treated and cryopreserved EAs. Within each line, percentage values followed by the same letter are not significantly different at the P $\leq 0.05$ by ANOVA and LSD test) 
1052
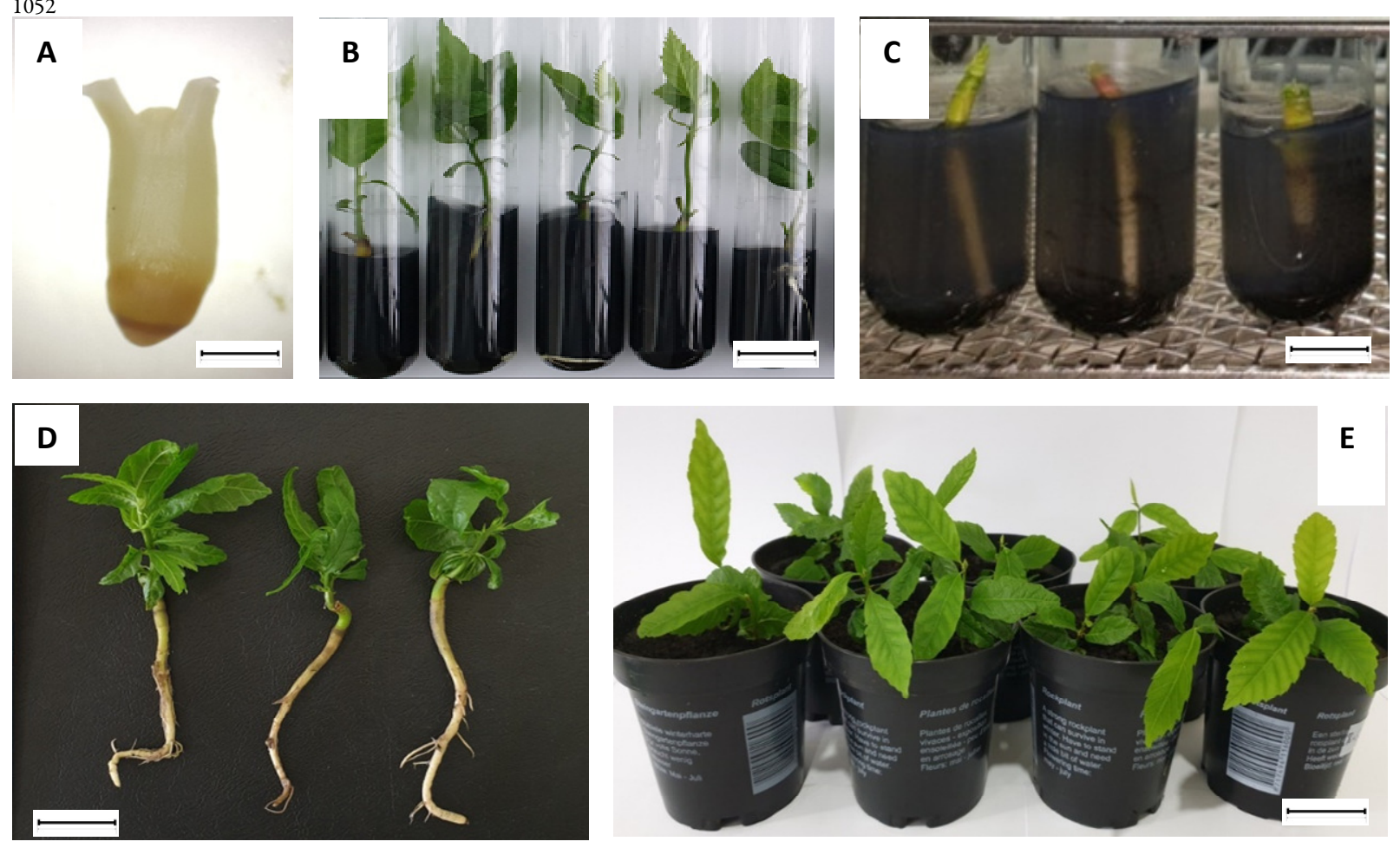

Fig. 5. Cryopreservation of Castanea sativa zygotic embryos: A) one embryonic axis (EA), along with cotyledonary knots and 2-3 mm-long cotyledon residuals, used for cryopreservation trials (bar, $1 \mathrm{~mm}$ ); B) initial plantlet development in post-cryopreservation by the dehydration/ 'one-step freezing' procedure, following a 5-h dehydration period of EAs, 2 weeks after re-culturing on WPM medium, supplemented with $0.3 \%$ activated charcoal (bar, $20 \mathrm{~mm}$ ); C) EAs that showed only root development and did not evolve further (bar, $10 \mathrm{~mm}$ ); D) plantlet regrowth in post-cryopreservation, 4 weeks after re-culturing in the aforesaid medium (bar, 20 $\mathrm{mm}$ ); E) plant development from cryopreserved EAs, 4 weeks after potting and acclimatization (bar, $50 \mathrm{~mm}$ )

tolerance to ultra-rapid freezing in $\mathrm{LN}$, such as in various conifers, while in many others a pre-dehydration to a $\mathrm{MC}$ around $20 \%$, followed by slow cooling or by direct immersion in LN, is strictly necessary (Panis and Lambardi, 2006). However, when embryos are composed by large cotyledons, as in chestnut, some insurmountable problems (i.e., the impossibility to insert them in cryovials, the large volume occupied in the dewar when using large boxes, the difficulties in inducing homogeneous dehydration or vitrification) induce to the use of excised EAs for cryopreservation. EAs, composed of the zygotic embryo and 2-3 $\mathrm{mm}$ of residual cotyledons, have been used for cryopreservation in this study. The presence of a small portion of cotyledons showed to be necessary to allow a germination, differently to what observed in others species, such as peanut (Ozudogru et al., 2009) where the EA totally prived of cotyledons does not evidence any negative effect during germination. On the opposite, every reduction of cotyledons compromises Pistacia vera seed germinability in post-cryopreservation (Ozden-Tokatly et al., 2007).

Physical dehydration and vitrification cryo-procedures, both allowing the direct immersion of specimen in LN ('one-step freezing'), have been applied to the cryopreservation of embryonic axes from temperate (Pence, 1990; Ozden-Tokatly et al., 2007; Ozudogru et al., 2009) and tropical plant species (Berjak and Dumet, 1996; Berjak et al., 1999). Physical dehydration is obtained, generally, exposing the EAs to the sterile air of a laminar flow hood for an appropriate time, adequate to minimize ice-crystal formation but, at the same time, high enough to avoid reaching a minimum level, below which damages for cell plasmolysis can be produced. This minimum level should be previously determined, and it can reach very low values. In Phaseolus vulgaris and Medicago sativa, for instance, seeds can be dehydrated to $7 \%$ MC, maintaining over $90 \%$ germinability after cryopreservation (Stanwood and Bass, 1981). Similarly, with peanut EAs, maximum germinability $(100 \%)$ was still obtained following the reduction of the initial MC from 25\% to 8.5\% (Ozudogru et al., 2009).

The natural MC of EAs of chestnut was $66.2 \%$, a value similar to what reported by other Authors (Pence, 1992; Correidora et al., 2004), and germinability was not affected up to $4 \mathrm{~h}$ dehydration (MC, 22.7\%), while showed a reduction to $84 \%$ of full germination (plantlet regrowth) after 5-h dehydration (MC, 21.4\%). Zygotic embryos of Correidora et al. (2004) had an initial survival of $83 \%$, and had a beneficial effect from the dehydration treatment (2-6 h) that induced a $100 \%$ survival. In this study, survival of EAs was always $100 \%$ and was not affected by the increasing time of dehydration treatment. In accordance with what observed by Correidora et al. (2004), also in the Georgian provenance of Castanea sativa the dehydration/'one-step freezing' procedure showed to be very effective in inducing a clear tolerance of EAs to ultra-rapid freezing in LN. Indeed, 
after $5 \mathrm{~h}$ dehydration, EAs achieved maximum survival in post-cryopreservation of $80 \%$, followed by a plantlet regrowth of $66.7 \%$. Besides the high germinability obtained with the dehydration/cryopreservation procedure, the germinated EAs could be easily acclimatized and produced healthy seedlings, without any sign of morphological alteration or callus proliferation. It is interesting to note that, in order to evaluate the efficacy of the cryo-procedure, it is not sufficient to evaluate the early germination (survival), but to determine the full germination. In Georgian chestnut plantlet regrowth (full germination) should be evaluated 4 weeks after thawing and culturing EAs in test tubes.

Also the PVS2-vitrification protocol developed in the present study showed a good applicability to the Georgian chestnut provenance, although to a lesser extent than the dehydration/'one-step freezing' technique. It is worthy of note that the incubation of specimens with a LS solution, prior to exposure to PVS2, was beneficial, as showed also from the partial survival and plantlet regrowth from EAs that had been only pre-treated in LS prior to cryopreservation. This is consistent with the general idea that a pre-loading of explants with cryoprotectants (at lower concentrations than they have in the vitrification solutions) is useful to minimize the possible adverse effects caused by the PVS2-vitrification solutions (Sakai, 2000). Indeed, the components of the cryoprotectant solution (glycerol, sucrose) are not permeable in the short period used for loading. However, such application causes cells to plasmolyze as result of considerable dehydration, and the presence of LS solution in the periprotoplasmic space of the plasmolyzed cells probably mitigates mechanical stress caused by severe dehydration due to exposure to PVS2 (Matsumoto et al., 1998).

The risk of toxicity of the vitrification solution is well known (Lambardi et al., 2000; Kim et al., 2011), and it is probably caused by the denaturation of proteins. The present study demonstrated that chestnut EAs tolerated well the PVS2 treatments in terms of early germinability (survival), but less in terms of further gemination (plantlet regrowth), particularly starting from 60-min treatment. As a consequence, the apparently low germinability obtained with the application of the PVS2 solution to EAs prior direct immersion in LN (with a maximum of plantlet regrowth of $55.6 \%$ following a 30-min treatment) should be interpreted as the best that could be achieved, considering the almost equivalent result achieved from EAs that had been equally treated but not frozen in LN.

It has been shown in this study that only a portion of the EAs graded 'survived' 7 days after dehydration/PVS2vitrification and immersion in $\mathrm{LN}$ were able to germinate afterwards, and 4-8 weeks had to be waited for a final evaluation of the tested cryo-procedures. Hence, in order to find a marker which could provide an early and reliable evaluation of seed germinability, the TTC test was used. The TTC test is a biochemical method, originally developed by Lakon (1949), in which viability is determined by the red colour appearing after soaking the seeds in a TTC solution for at least overnight, depending on the species. Living tissue changes the TTC to an insoluble red compound (formazan); in non-living tissue the TTC remains uncoloured. The grading of a seed into "viable" or "nonviable" depends upon the amount and location of white areas. As a consequence, correct interpretation depends upon standards worked out for specific seeds (MacKay, 1972). When the EAs of the Georgian provenance of
Castanea sativa were tested just after their recovery from $\mathrm{LN}$, a good correlation with their germinability was found for seeds specimen, showing completely red embryos and residual cotyledons. The general overestimation which was observed for both dehydrated/LN+ and vitrified/LN+ (starting from 30-min exposure to PVS2) EAs is in accordance with what reported, e.g., in Pistacia spp. seeds (Ozudogru et al., 2009).

\section{Conclusions}

In conclusion, the present study has clearly demonstrated that the application of both dehydration and vitrification systems for cryopreservation of chestnut zygotic embryos resulted in viable embryos capable to develop fully developed plantlets. Dehydration gave better EAs survival and plantlet regrowth, as well as a more rapid organized plant development after cryopreservation, thus proving to be more effective practice in compare to the PVS2vitrification/'one-step freezing' technique. However, this lower performance of the latter procedure has been shown to be due to an intrinsic toxic effect of the PVS2 solution on the treated chestnut zygotic embryos. Research in this field should move in the direction to test for chestnut zygotic embryos other less toxic vitrification solutions (Kim et al., 2011).

\section{Acknowledgements}

This work was supported by Shota Rustaveli National Science Foundation of Georgia (SRNSFG), grant number $04 / 52$, and by the National Research Council of Italy, in the frame of the CNR-SRNSF Bilateral Project "Developing efficient cryopreservation procedures for the long-term storage of endangered plant genetic resources of Georgia". Carla Benelli and Maurizio Lambardi thank also the contribution of the "International Treaty on Plant Genetic Resources for Food and Agriculture/RGV-FAO, 20172019”.

\section{Conflict of Interest}

The authors declare that there are no conflicts of interest related to this article.

\section{References}

Barstow M, Khela S (2018). Castanea sativa. The IUCN Red List of Threatened Species, e.T202 948A67740523 from http:// dx.doi.org/ 10.2305/IUCN.UK.2018-1.RLTS.T202948A67740523.en.

Benelli C, De Carlo A, Engelmann F (2013). Recent advances in the cryopreservation of shoot-derived germplasm of economically important fruit trees of Actinidia, Diospyros, Malus, Olea, Prunus, Pyrus and Vitis. Biotechnology Advances 31(2):175-185.

Berjak P, Dumet D (1996). Cryopreservation of seeds and isolated embryonic axes of neem (Azadirachta indica). CryoLetters 17:99-104.

Berjak P, Pammenter NW (1997). Progress in the understanding and manipulation of desiccation-sensitive (recalcitrant) seeds. Basic and Applied Aspects of Seed Biology 30:689-703.

Berjak P, Walker M, Watt MP, Mycock DJ (1999). Experimental parameters underlying failure or success in plant germplasm 
1054

cryopreservation: A case study on zygotic axes of Quercus robur L. CryoLetters 20:251-262

Capuana M, Di Lonardo S (2013). In vitro conservation of chestnut (Castanea sativa) by slow growth. In Vitro Cellular \& Developmental Biology-Plant 49(5):605-610.

Conedera M, Jermini M, Sassella A, Sieber TN (2004). Récolte, traitement et conservation des châtaignes [Collection, treatment and conservation of chestnut fruits]. Notice pour le praticien, WSL, Birmensdorf 38:1-12.

Corredoira E, San-Jose MC, Ballester A, Vieitez AM (2004). Cryopreservation of zygotic embryo axes and somatic embryos of European chestnut. CryoLetters 25:33-42.

Gagnidze R (1975). Fagaceae. In: Ketskhoveli N, Kharadze A, Gagnidze R (Eds). Sakartvelos flora [Flora of Georgia], 2nd ed, vol III, Metsniereba, Tbilisi, pp 40-43.

Giacalone G, Bounous G (1993). Tradizioni ed innovazioni nella trasformazione enell'utilizzo delle castagne [Tradition and innovation in the transformation and utilization of chestnut fruits]. Monti Boschi 44(5):33-41.

Gulisashvili VZ (1961). Dendroflora Kavkaza [Dendroflora of the Caucasus]. Izdatelstvo AN GSSR, Tbilisi.

IUCN Red List of Threatened Species (2019). IUCN Red List version 2019-1, Sweet Chestnut (Castanea sativa) from https://www.iucnredlist.org/species/202948/67740523.

Kim HH, No NY, Shin DJ, Ko HC, Kang JH, Cho EG, Engelmann F (2011). Acta Horticulturae 908:181-186.

Lakon G (1949). The topographical Tetrazolium method for determining the germinating capacity of seeds. Plant Physiology 24(3):389-394.

Lambardi M, Fabbri A, Caccavale A (2000). Cryopreservation of white poplar (Populus alba L.) by vitrification of in vitro-grown shoot tips. Plant Cell Reports 19(3):213-218.

Lambardi M, Benelli C, De Paoli G, Battistini A (2001). Biotecnologie per la Conservazione del Castagno [Biotechnology for the conservation of chestnut]. Proc. "Convegno Nazionale Castagno 2001". Marradi (Firenze), 25-27 October, pp 86-91.

Leprince O, Buitink J, Hoekstra FA (1999). Axes and cotyledons of recalcitrant seeds of Castanea sativa Mill. exhibit contrasting responses of respiration to drying in relation to desiccation sensitivity. Journal of Experimental Botany 50(338):1515-1524.

Lloyd G, McCown BH (1981). Woody plant medium (WPM)-a mineral nutrient formulation for microculture of woody plant species. HortScience 16:453-453.

MacKay DB (1972). The measurement of viability. In: Roberts EH (Ed). Viability of seeds. Syracuse University Press, NY,pp 172-208.

Matsumoto T, Sakai A, Nako Y (1998). A novel preculturing for enhancing the survival of in vitro-grown meristems of wasabi (Wasabia japonica) cooled to $-196^{\circ} \mathrm{C}$ by vitrification. CryoLetters 19(1):27-36.

Nakhutsrishvili G (2013). Forest vegetation of Georgia. In: Nakhutsrishvili $\mathrm{G}(\mathrm{Ed})$. The vegetation of Georgia (South Caucasus). Springer, pp 3587.

Normah MN, Makeen AM (2008). Cryopreservation of excised embryos and embryonic axes. In: Reed BM (Ed). Plant cryopreservation. A practical guide. Springer, New York, pp 211-240.

Ozden-Tokatli Y, Ozudogru EA, Gumusel F, Lambardi M (2007). Cryopreservation of Pistacia spp. seeds by dehydration and one-step freezing. CryoLetters 28(2):83-94.
Ozudogru EA, Ozden-Tokatli Y, Gumusel F, Benelli C, Lambardi M (2009). Development of a cryopreservation procedure for peanut (Arachis hypogaea L.) embryonic axes and its application to local Turkish germplasm. Advances in Horticultural Science 23(1):41-48.

Panis B, Lambardi M (2006). Status of cryopreservation technologies in plants (crops and forest trees). The role of biotechnology in exploring and protecting agricultural genetic resources. FAO, Rome, pp 61-78.

Pence VC (1990). Cryostorage of embryo axes of several large-seeded temperate tree species. Cryobiology 27(2):212-218.

Pence VC (1992). Desiccation and survival of Aesculus, Castanea and Quercus embryo axes through cryopreservation. Cryobiology 29(3):391399.

Pritchard HW, Nadarajan J (2008). Cryopreservation of orthodox (desiccation tolerant) seeds. In: Reed BM (Ed). Plant cryopreservation. A practical guide. Springer, New York, pp 485-501.

Pridnya MV, Cherpakov VV, Paillet FL (1996). Ecology and pathology of European chestnut (Castanea sativa) in the deciduous forests of the Caucasus Mountains in southern Russia. Bulletin of the Torrey Botanical Club 123(3):213-222.

Red List of Georgia (2006). The decree of the president of Georgia on approval of the Red List of Georgia. Tbilisi from: https:// www.matsne.gov.ge/ka/document/view/97288?publication=0

Reed BM (2008). Plant cryopreservation. A practical guide. Springer, New York.

Sakai A, Kobayashi S, Oiyama I (1990). Cryopreservation of nucellar cells of navel orange (Citrus sinensis Osb. var. brasiliensis Tanaka) by vitrification. Plant Cell Reports 9(1):30-33.

Sakai A (2000). Development of cryopreservation techniques. In: Engelmann, F and Takagi H (Eds.). Cryopreservation of tropical plant germplasm. Current research progress and application. IPGRI, Rome, Italy, pp 1-7.

San-José MC, Jorquera L, Vidal N, Corredoira E, Sánchez C (2005). Cryopreservation of European chestnut germplasm. In: III International Chestnut Congress 693, pp 225-232.

Stanwood PC, Bass LN (1981). Seed germplasm preservation using liquid nitrogen. Seed Science and Technology 9:423.

Tavadze B, Supatashvili A, Kapanadze G, Mamukashvili TS (2012). Pathological status of chestnut stands in Tkibuli region (Georgia). Annals of Forestry 5:21-32.

Vertucci CW, Farrant JM (1995). Acquisition of desiccation tolerance. In: Kigel J, Galili G (Eds). Seed development and germination. Marcel Dekker Press Inc., New York, pp 237-271.

Vidal N, Sanchez C, Jorquera L, Ballester A, Vieitez AM (2005). Cryopreservation of chestnut by vitrification of in vitro-grown shoot tips. In Vitro Cellular \& Developmental Biology-Plant 41(1):63-68.

Vidal N, Vieitez AM, Fernandez MR, Cuenca B, Ballester A (2010). Establishment of cryopreserved gene banks of European chestnut and cork oak. European Journal of Forest Research 129(4):635-643.

Walters C, Wesley-Smith J, Crane J, Hill LM, Chmielarz P, Pammenter NW, Berjak P (2013). Cryopreservation of recalcitrant (i.e. desiccationsensitive) seeds. In: Reed BM (Ed). Plant cryopreservation. A practical guide. Springer, New York, pp 465-484.

Westengen OT, Jeppson S, Guarino L (2013). Global ex-situ crop diversity conservation and the Svalbard global seed vault: assessing the current status. PloSOne 8(5):e64146. 